\title{
Approches(s) anthropologique(s) en éducation et en formation, enjeux et défis
}

Marie-Louise Martinez-Verdier

\section{(2) OpenEdition}

\section{Journals}

Édition électronique

URL : http://journals.openedition.org/trema/530

DOI : 10.4000/trema.530

ISSN : 2107-0997

Éditeur

Faculté d'Éducation de l'université de Montpellier

Édition imprimée

Date de publication : 1 mai 2004

Pagination : $3-24$

ISSN : 1167-315X

\section{Référence électronique}

Marie-Louise Martinez-Verdier, «Approches(s) anthropologique(s) en éducation et en formation, enjeux et défis », Tréma [En ligne], 23 | 2004, mis en ligne le 05 octobre 2010, consulté le 21 septembre 2020. URL : http://journals.openedition.org/trema/530; DOI : https://doi.org/10.4000/trema.530

Ce document a été généré automatiquement le 21 septembre 2020

Trema 


\title{
Approches(s) anthropologique(s) en éducation et en formation, enjeux et défis
}

\author{
Marie-Louise Martinez-Verdier
}

\begin{abstract}
« La science de l'homme, parvenue à un certain accomplissement, se doit de livrer l'idée de l'homme qui est impliquée par sa démarche et par ses résultats, mais qui est laissée, pour l'essentiel, à l'état implicite. Ce dévoilement est nécessaire, à la fois pour mieux faire la science et pour la faire mieux comprendre et accepter.» Pierre Bourdieu ${ }^{1}$
\end{abstract}

\section{Problématique}

1 Comment relier le sens avec les savoirs? Comment articuler le souci du sujet singulier et de son développement avec celui du bien commun (scientifique, économique, social, culturel, politique ...) enfin avec celui de l'Homme ? Comment former des enseignants à la hauteur des exigences d'une profession de plus en plus complexe: attentifs à la diversité des singularités et efficaces dans l'instauration d'une solidarité citoyenne et démocratique, au cœur de la transmission des savoirs?

2 L'anthropologie dans ses différents courants scientifiques ou philosophiques est caractérisée par un questionnement sur le propre de l'humain (anthropos: l'homme) dans et malgré sa diversité culturelle. Elle se définit souvent aujourd'hui par le souci de comprendre le mouvement unique qui permet à l'individu de s'humaniser en s'affiliant au groupe et, par là-même, devenir sujet dans son unicité.

3 L'anthropologie a pris naissance deux fois, d'abord comme philosophie puis, bien plus tard, comme science sociale. Aussi ancienne que la philosophie, la question du propre de l'homme se fait plus précise dans celle du sujet. L'anthropologie philosophique ${ }^{2}$ 
aujourd'hui se concentre sur le lien entre la singularité subjective et l'altérité vers la réflexivité universelle. Cette aspiration dans la philosophie occidentale est présente déjà depuis Kant mais, depuis Hegel, elle fait chaque fois plus de place à la relativisation du sujet.

L'anthropologie comme science, est plus récente: même si on peut lui trouver de lointains précurseurs chez le grec Hérodote ou le latin Tacite, elle naît à la fin du XVIIIe siècle avec l'étude des sociétés archaïques. Au départ des sciences sociales ou sciences de l'homme, l'anthropologie et la sociologie se développent de manière jumelle, l'une se consacre à l'étude des sociétés dites primitives, homogènes et holistiques, tandis que l'autre se voue à celles des sociétés complexes et hétérogènes. Aujourd'hui, toutes deux convergent dans leurs objets et elles ont souvent en commun aussi la recherche de structures et de processus, comme par exemple les phénomènes rituels ou ceux de l'échange symbolique ${ }^{3}$. Autrefois limité à l'étude du primitif qui n'existe plus, le discours de l'anthropologie se tourne vers un retour sur soi et sur l'autre considérés ensemble : un regard sur soi tributaire de l'existence de l'autre.

L'approche anthropologique, au sens large, tant philosophique que scientifique trouve son unité dans le fait qu'elle est attentive au lien entre la singularité particulière et les processus relationnels, vers la question de l'universel. Elle diffère dans les questionnements et les démarches méthodologiques.

6 L'anthropologie philosophique questionne les fondements et utilise l'analyse critique et conceptuelle. L'anthropologie scientifique, quant à elle, c'est plutôt les fondations qu'elle interroge jusque dans les fonctionnements du quotidien. Plus expérimentale, elle demande le recueil et l'analyse de données sur les terrains particuliers pour les interpréter dans un modèle plus général. L'anthropologie scientifique parvient au niveau de généralité ou mieux d'universalité auquel elle aspire, grâce aux démarches et méthodes qu'elle peut conjuguer naturellement par le système des sous-disciplines qui la composent: ethnographie, ethnologie et anthropologie (au sens plus précis). Elle peut ainsi aller de la description ethnographique à l'élaboration d'hypothèses plus générales dans le moment ethnologique vers l'établissement de modèles plus fondamentaux dans l'anthropologie générale, ces trois temps peuvent s'emboîter de manière hiérarchique selon les étapes ou les ancrages de la recherche. Ainsi, sans se confondre dans leurs questionnements ni leurs démarches, l'anthropologie sociale et l'anthropologie philosophique peuvent se rejoindre et s'enrichir réciproquement.

7 Les atouts: des démarches articulables (3 niveaux hiérarchisés d'analyse) une compatibilité des problématiques et des concepts permettant aux disciplines qui se tournent vers l'approche anthropologique d'envisager de travailler ensemble en un véritable paradigme.

Longtemps repoussée, voire tabou ${ }^{4}$, en sciences de l'éducation, l'approche anthropologique dans la gamme de ses variations est aujourd'hui au goût du jour: de nombreux colloques l'affichent, des numéros de revue s'y consacrent, de plus en plus de recherches et de publications s'y réfèrent. 
9 Loin de devoir être considéré comme un simple et éphémère effet de mode, le mouvement est probablement plus profond, il répond à des défis importants sur le plan social, institutionnel, épistémologique et théorique. Sans menacer d'une intrusion globalisante qui viendrait dans ses prétentions holistiques, absorber les contours et les spécificités des diverses disciplines des sciences de l'éducation (philosophie de l'éducation, sociologie, psychologie, psychologie sociale de l'apprentissage, didactique des disciplines, histoire, etc.) l'approche anthropologique leur offre plutôt un espace de convergence et de synergie. On assiste à une sorte de tournant pris par diverses disciplines qui leur permettraient une rencontre nouvelle dans la complémentarité, sans leur faire perdre leur pertinence, sans effacer la légitimité d'un travail épistémologique spécialisé sur l'éducation. De même qu'on a pu parler en d'autres temps d'un linguistic turn, d'un "tournant linguistique» en sciences humaines, on pourrait parler aujourd'hui de "tournant anthropologique ». Il correspond à un style d'interrogation et à un mode de questionnement nouveau : œil étonné ouvert sur les fonctionnements dans la boîte noire (ethnographie de l'éducation), regard tourné vers les fondations (anthropologie scientifique ou générale), redéfinition des fondements dans leur implication (anthropologie philosophique) : le sujet, les finalités, les valeurs de l'éducation ...

Quand le propre et le familier ne vont plus de soi, il convient de porter sur eux le regard de l'altérité du huron, de considérer leur étrangeté, de mesurer leur pesant de particularismes voire d'arbitraire. Cette critique, commencée depuis le XVIe siècle pour nos sociétés, est sommée aujourd'hui de s'accélérer. Nos sociétés plurielles, appelées chaque fois plus à se confronter à l'international, à l'universel, doivent revisiter les fondements de leurs institutions et redéfinir les projets en fonction du choix assumé d'une humanité commune et multiple. Sont appelées à y travailler les sciences humaines dans leur ensemble, non seulement l'ethnographie, l'ethnologie et l'anthropologie, elles-mêmes mais les nombreuses approches anthropologiques jaillies au cœur ou à la périphérie d'autres disciplines comme l'histoire, la linguistique, la psychologie, la sociologie, la didactique, etc.

11 La réflexion en éducation et en formation, en prenant ce tournant scientifique peut bénéficier aujourd'hui de l'émergence d'un véritable paradigme ${ }^{5}$ en sciences humaines et sociales. Le paradigme permet la compatibilité et l'articulation épistémologique entre les principaux concepts et modèles sans jamais indifférencier les discours ni effacer les précieuses particularités disciplinaires.

Le rendez-vous avec le tournant anthropologique et ses diverses approches était d'importance pour l'étude de l'homo éducans ${ }^{6}$ dans son élan d'humanisation, de socialisation et de subjectivation.

\section{Les enjeux d'une approche anthropologique}

Dans la conjoncture actuelle, sur divers plans (social, institutionnel et scientifique) de nombreux aspects sont en jeu et posent problème à l'école de façon aiguë. Devant ces difficultés, les démarches et les approches habituelles sont en crise entraînant alors des résistances, dans l'action et la connaissance. Mais ce qui était insurmontable pour certains cadres théoriques devient possible aux nouvelles approches qui s'expérimentent et se confirment dans ce nouveau contexte. 

au centre du système éducatif ». L'expression en elle-même au fond dit bien l'essentiel (il y est question de l'élève, pas de l'enfant !) mais elle est grosse de malentendus sur des aspects sensibles : l'individualisme exacerbé, la crise de la transmission et le difficile rapport adulte-enfant. Porteur d'une conception puerocentrique, le terme s'est chargé de connotations laxistes et démissionnaires, dans le contexte civilisationnel qui est le nôtre et dont la philosophe Hannah Arendt a su montrer qu'il était surtout crise de l'éducation et de l'autorité. Philippe Ariès, dans ses travaux ${ }^{9}$ sur les avatars de l'enfance et de l'adolescence au XXe siècle n'avait-il pas montré comment, au centre de toutes les attentions et de toutes les permissivités, cette posture royale devenait place de la victime. L'enfant roi sans recours éducatif se retrouve enfant proie de lui-même comme de ses ambivalents adorateurs. Telle quelle, l'expression peut charrier des confusions et escamoter tout rapport contraignant et structurant au savoir en absorbant l'instruction dans une sirupeuse convivialité "socio-animatrice». Revendiquer la centralité de l'enfant sans autres précautions était alors une maladresse et une provocation dont les contempteurs de la pensée soixante-huit se sont saisis avec une évidente mauvaise foi. Autour de cela, se sont déchaînées de vaines joutes oratoires entre les partisans d'une 
didactique qui effaçait le rôle de la relation interpersonnelle et ceux d'une pédagogie qui minimisait l'impact des savoirs au profit d'un « épanouissement » de l'enfant.

Ces oppositions simplistes, manichéennes et stériles faisaient toujours l'impasse sur l'essentiel : un travail conceptuel d'analyse des puissants phénomènes relationnels qui croisent et articulent l'intersubjectif aux savoirs avec leurs objets et leurs genres. L'enjeu est énorme, il concerne la question du sujet de l'éducation : le sujet singulier qui émerge dans, par et pour la relation d'apprentissage-enseignement. Les malentendus individualistes se dissipent autour du défi de comprendre l'émergence de l'élève, comme sujet (ou mieux comme personne) grâce à une relation éducative structurante avec l'adulte enseignant, dans et par les interactions que permettent les genres discursifs de l'école et des disciplines du savoir. Il s'agit de mettre en œuvre une relation éducative, au sens non trivial du terme, pour permettre à l'élève d'émerger comme sujet dans et par l'investissement et l'appropriation des savoirs, dans et par la relation au groupe de pairs où se construit une socialisation juste et citoyenne. Relation médiatisée par les notions, les pratiques et les genres discursifs des savoirs selon les spécificités des diverses disciplines.

Se retrouvent alors les classiques finalités, définies par l'anthropologie philosophique de Kant $^{10}$ (instruction et socialisation) croisées et réconciliées entre elles et surtout avec la fin de l'éducation: émergence de l'élève comme sujet réflexif, un parmi d'autres, soucieux d'autrui autant que de soi-même c'est-à-dire personne $e^{11}$.

La compréhension plus subtile du sujet de l'éducation et de l'identification de l'élève comme sujet et personne ${ }^{12}$, par l'impact des interactions langagières au cœur des pratiques scolaires avec leurs genres disciplinaires selon la dynamique intersubjective de la relation éducative, demande un lourd travail théorique. Voilà un des défis majeurs de la formation d'enseignants que pourraient relever différentes variétés d'approches anthropologiques.

21 Le second enjeu, lié au premier, touche à l'articulation difficile des identités disciplinaires de l'éducation et de la formation. Comment, en effet, comprendre ensemble les traits communs et les différences entre les divers savoirs académiques enseignés? Quelles sont les caractéristiques spécifiques des disciplines, quelles complémentarités ont-elles dans le vaste prisme de l'épistémologie des savoirs scolaires? Chaque tradition de spécialistes jusque là, vivait dans une planète ou une forteresse distincte avec sa culture, ses traditions, ses mythes fondateurs et ses pratiques. Mais quelle transversalité partager, quelles notions et concepts communs pour harmoniser les analyses de pratiques, entre les didacticiens des disciplines, quel agir communicationnel éducatif convergent?

Quelles culture commune et spécificités structurantes entre le primaire, le secondaire et le technique? Comment démanteler les cloisonnements entre les niveaux d'enseignement comme à l'intérieur du collège et du lycée ? Comment articuler et instaurer une transversalité bienfaisante sans indifférencier les niveaux, les discours ni les savoirs? Malgré le chemin parcouru grâce à l'apport des didactiques, et plus récemment grâce à la question du français transversal, les cloisonnements demeurent. Ils verrouillent l'incommunicabilité entre les didacticiens des disciplines qui sont pourtant appelés à coopérer dans la formation de généralistes du primaire. L'enjeu 
dépasse celui d'une simple convivialité dans les salles de profs, il demande de construire les références communes d'une professionnalité enseignante, selon les disciplines et les niveaux d'enseignement dans le système scolaire. Il est de taille non seulement pour l'identité enseignante (une même profession dans ses diversités), mais pour l'élève (cohérence dans le rapport aux savoirs, à l'école et à la culture).

Il faut pour cela comprendre et dire dans des termes à la fois communs et spécifiques, ces processus complexes d'intersubjectivation au cœur des savoirs enseignés par les différentes disciplines, selon leurs pratiques spécifiques et les genres discursifs qui les définissent. Car c'est bien dans les rituels interlocutifs des genres disciplinaires et scolaires que s'élabore l'appropriation des savoirs et des connaissances, que s'opère la socialisation des élèves, que s'affine la définition du sujet.

tablissant des ponts et des liens entre les divers concepts et pratiques des disciplines, en saisissant les spécificités, au lieu d'être les spectateurs captifs de manières de dire et de faire hétérogènes et arbitraires, les élèves pourraient participer plus créativement à leur propre processus d'apprentissage.

On attend les IUFM sur cet objectif d'une culture commune mais sans confusion indifférenciatrice entre le primaire, le secondaire et le technique, entre les diverses disciplines et la transversalité éducative : elle s'invente aujourd'hui, mais on tarde à en constater avec évidence le bilan positif. Les approches anthropologiques sont bien outillées pour aider les IUFM à relever ce défi.

Enfin, toujours lié aux deux précédents, le troisième enjeu touchant à la vocation propre des IUFM, c'est bien sûr, celui de la professionnalisation des métiers de l'enseignement.

En quoi, une institution spécifique, avec des lieux et une histoire commune, peut-elle apporter une pierre décisive dans l'identité des métiers de l'enseignement et de la formation, par rapport à l'Université d'une part et la formation sur les terrains de l'école d'autre part? Comment relier en articulant, en tressant finement dans une métabolisation singulière et réflexive, pour chaque enseignant, le rapport entre théorie et pratique ? C'est sans doute ce lieu géométrique d'une conjonction problématique qui constitue le défi majeur des IUFM. Vont-ils savoir permettre par l'alternance formative cette intégration de contenus théoriques exigeants avec la gestualité pratique? Par quelles démarches et quelles approches concentrer le retour réflexif au cœur du processus d'identification professionnelle?

C'est probablement là, précisément dans cette nécessité de nouer la particularité et la diversité des «terrains » au cœur d'une réflexion plus générale ou fondamentale, pour un sujet singulier et réflexif (qui a le souci de soi, de l'autre et de l'institution juste (Ricœur) que l'anthropologie avec ses approches et ses démarches spécifiques est directement interpellée. Elle pourra d'ailleurs, sur ce point, croiser les références à la pensée de l'anthropologie philosophique sur le soi (Kant, Ricœur) et à l'ethnographie de l'école (P. Woods), les travaux de Philippe Perrenoud ${ }^{13}$ sont ici exemplaires. 
I.3. Les enjeux épistémologiques et méthodologiques sont très prégnants aussi. Dans la cité des sciences sociales et humaines, et surtout dans ses carrefours épistémologiques les plus féconds comme les sciences de l'éducation ou les sciences criminelles, où elles sont convoquées à coopérer activement et efficacement, les diverses disciplines ne font pas toujours bon ménage. En fonction de leur histoire et de leurs institutions, les sciences comme la psychologie, la médecine, la sociologie, l'histoire, la linguistique, l'économie, entre elles ou avec la philosophie ou encore avec les diverses disciplines d'enseignement (littérature, mathématiques, etc.) ont de lourds contentieux de territoire. Elles redoutent, sans doute à juste titre, les tentations hégémoniques et globalisantes qui viendraient défigurer la coopération dans le respect des frontières, avec de graves conséquences sur la répartition des professions dans les différentes institutions du système scolaire ou même du travail social où se livrent d'impitoyables guerres d'influence et de concurrence en ces temps de crise des différences. La méfiance des sciences de l'éducation à l'encontre de la philosophie, discipline mère, d'où la plupart des disciplines sont issues est aussi très sensible. De leur émancipation scientifique, souvent marquée par de tapageuses ruptures épistémologiques, elles gardent les stigmates d'une naissance et d'une croissance batailleuse et se tiennent à distance craignant inféodation ou allégeance. Dans le meilleur des cas, elles se regardent en chiens de faïence, crispées sur un vague consensus de pluri-référentialité qui ne favorise pas toujours la rigueur méthodologique. Le pacte suspicieux de non-agression réciproque sur lequel elles campent n'autorise pas toujours la saine collaboration voire l'intégration épistémologique que les enjeux sociaux et institutionnels réclament aujourd'hui.

Le besoin d'une réflexion fondamentale sur l'épistémologie des disciplines et les effets sur les savoirs, le sujet, le lien social, sur l'éthique, se fait ressentir avec celui d'une articulation entre les questions. Pour refonder le sujet de l'éducation, penser l'humain dans l'homme et lier cette humanisation (comme processus singulier et collectif) à la question du sens, il faut articuler dans l'éducation la question du développement du sujet, de la socialisation dans le groupe, à celle des spécificités des grands champs symboliques des savoirs et de leurs genres.

31 Pour cela, il faut lever le verrou positiviste présent encore dans certaines disciplines qui ne peuvent pas penser le sujet. Mais il faut aussi éviter de cliver ces questions dans les découpages habituels du travail théorique : le sujet confisqué par la psychanalyse ou la linguistique, le groupe par la sociologie ou la psychologie-sociale et l'humanisation cantonnée dans la paléontologie ou la philosophie. Coûteuse fragmentation qui empêche de penser ensemble ces mouvements interfaces, d'articuler ces niveaux. Cela demande des approches et des démarches interdisciplinaires et transdisciplinaires plus souples et compatibles entre elles.

32 Les approches anthropologiques qui se sont développées ces dernières décennies aux confins de chaque discipline (l'ethnométhodologie, l'ethnopsychiatrie, l'anthropologie sociale, l'interactionnisme symbolique qui se concentre sur les interactions sociales comme microrituels, l'ethnolinguistique conversationnelle, l'ethnohistoire ou la microhistoire du quotidien, la philosophie pragmatique de l'interlocution, entre autres fleurons d'un vaste paradigme) peuvent converger en ce sens.

En éducation et en formation, elles actualisent et opérationnalisent ce mouvement par la mise en oeuvre de concepts, de modèles et de démarches carrefours. L'anthropologie présente l'avantage méthodologique d'avoir su articuler des démarches empiriques, 
descriptives et inductives ou même déductives (à partir de modèles), comme étapes ou moments complémentaires de la recherche dans des processus plus vastes. En cette aptitude théorique et méthodologique aussi, elle offre des ressources inespérées aux sciences de l'éducation en adéquation avec les défis actuels.

Comment alors comprendre les résistances et les rejets qui ont été opposés?

\section{Les résistances}

Au-delà des triviales défenses territoriales dans la cité épistémique des sciences de l'éducation de la part de rivales menacées, les rejets de l'approche anthropologique expriment des malaises et des tabous plus graves. Les résistances sont nombreuses et pugnaces, nous n'en développerons ici que trois aux conséquences particulièrement stérilisantes.

\section{II.1. Le tabou du sacré et du rite}

On ne peut pas étudier la question de l'identité, de l'appartenance communautaire, de la violence et de l'exclusion, du sens lui-même, en faisant abstraction de la question du sacré avec celle de rites, interdits et jeux qui en constituent la réalité.

Or les sciences de l'éducation refoulent ${ }^{14}$ cette étude.

La question du sacré et du rite a toujours été capturée par la pensée religieuse, confinée dans les disciplines théologiques et confisquée par une caste cléricale, dans une réserve prudente et méfiante par rapport aux outils de rationalité des sciences sociales, toujours susceptibles de sacrilège. Cela est regrettable mais facilement compréhensible. Mais curieusement, la même mise à l'écart méticuleuse du sacré dans un enclos spécifique est tout aussi grande sinon plus, de la part des sciences humaines modernes qui redoutent non seulement un retour intempestif du refoulé, mais peut-être surtout les paradoxes, les ambivalences, les retournements de la notion qu'elles se sentent incapables d'assumer avec les lourds outillages théoriques habituels. L'étude du sacré dans les sciences sociales a été cantonnée à l'étude des institutions religieuses uniquement dans leurs spécificités cultuelles.

Pourtant l'anthropologie du sacré avait pris un essor prometteur avec Durkheim et son neveu Mauss, chez qui elle dépassait largement les stricts objets religieux, autorisant des perspectives très heuristiques sur toutes les institutions. Après eux, Dumont, Girard, Goffman, etc. nous montrent depuis longtemps l'omniprésence du système sacrificiel dans toutes les institutions du social et pas seulement celles qui se consacrent au religieux. Celui-ci est constitué de rites, d'interdits, de jeux et de mythes, qui sous des formes diverses, religieuses ou profanes, ont pour fonction de réguler les tensions et les conflits endémiques entre les humains et de créer et recréer du lien social. Pour Durkheim ${ }^{15}$, le sacré est la réalité même du social, le lien social et ses institutions sont (ré) générés par le système rituel fait de prescriptions et proscriptions. Mauss confirme : «A notre avis est conçu comme sacré tout ce qui pour le groupe et pour ses membres qualifie la société ... $»^{16}$.

Non seulement le refus d'étudier le sacré dans les diverses institutions du social et particulièrement l'école est un gâchis heuristique et rationnel, mais en outre il a des 
conséquences redoutables sur le plan social. Quand le sacré n'est pas vu ni compris dans sa fonction de genèse du lien social, des identités communautaires et personnelles, et surtout de régulation de la violence, on assiste à deux processus qui s'engendrent et qui s'emballent réciproquement : une déritualisation anomique et une resacralisation ségrégative du social. Ces deux mouvements se constatent actuellement et ils forment les deux faces alternatives de la violence et du sacré.

Cette scotomisation obstinée stimule la prolifération d'une violence anomique, indifférenciatrice qui provoque des réactions d'identitarismes vindicatifs et de ségrégation ${ }^{17}$ sociale. On pourrait mieux comprendre les processus et peut-être envisager des alternatives grâce à certaines approches anthropologiques.

II.2. Les résistances à penser la conjonction entre la genèse des savoirs cognitifs, académiques et la genèse sociale ne sont pas moins nocives. Une certaine tradition scolastique s'entête à opposer l'instruction et l'éducation, de nombreux discours aujourd'hui s'obstinent à dresser les tenants de la didactique des savoirs contre ceux de la pédagogie comme souci transversal de la relation. Pour le linguiste Milner ${ }^{18}$ déjà, il ne fallait pas confondre les contenus et le contenant (ne pas prendre le moule de la pédagogie pour la pâte substantielle des savoirs). Le couplet est rejoué à chaque rentrée universitaire dans des pamphlets qui s'ingénient avec plus ou moins de bonheur à dresser des murs entre les connaissances académiques et la relation trop hâtivement comprise dans un sens banal de convivialité.

Enracinée dans de vieilles querelles catégorielles entre les représentants de l'école primaire (instituteurs généralistes soucieux d'instituer les enfants au sens que Montaigne déjà pouvait donner à l'expression) et ceux du secondaire (spécialistes des savoirs des matières et disciplines académiques), cette fracture idéologique, relayée par des modèles inadéquats de la communication (inspirés des télécommunications) est aujourd'hui devenue un redoutable obstacle épistémologique. Ce point aveugle empêche totalement de comprendre la complexité de l'enseignement : instaurer la paix sociale dans la classe, permettre à l'élève de construire la règle comportementale voire morale n'est pas du temps perdu sur la clarification cognitive et les apprentissages académiques, bien au contraire ! Enseigner c'est (étym.) faire entrer dans les signes et les significations symboliques qui sont faites de règles cognitives et sociales entretissées. Certaines approches anthropologiques permettent de comprendre cette compénétration de la genèse cognitive et sociale.

II.3. L'incapacité à prendre toute la dimension du sujet et de son émergence dans la relation interpersonnelle est un frein pour la compréhension de la véritable portée de l'éducation et de la formation.

Le positivisme en sciences sociales ignore ou refuse le sujet, pour lui il n'y a de science que d'objets observables et mesurables. On sait quels dégâts cette "mal mesure de l'homme ", cette catégorisation réifiante et disqualifiante, ont faits dans l'éducation, la psychiatrie ou les sciences sociales en général. L'enseignant et le formateur ne peuvent évaluer l'élève ni la situation éducative en toute extériorité, ils font eux-mêmes partie 
du problème. Le chercheur, lui-même sujet, ne peut étudier l'autre ni les autres comme des objets manipulables. Ils sont sujets pris ensemble dans l'intersubjectivation désirante pour construire des significations, définir et instituer ensemble le réel et le symbolique. Ils émergent ensemble dans cette production de savoirs sur l'homme, pour le meilleur et pour le pire, dans la stigmatisation ou la personnification réciproque.

Mais s'il faut critiquer le positivisme, il faut tout autant sortir d'une conception idéaliste du sujet comme pure intériorité destinée à "s'épanouir » dans l'expressivité ... Si le sujet a un socle biologique, physiologique, corporel, affectif et libidinal il n'est pas entité immédiate et émotionnelle qu'il s'agirait de libérer dans sa pure spontanéité des contraintes institutionnelles et sociales. Enfin, si le sujet ne peut se connaître ni connaître l'autre par une étude qui le chosifie et le réduit à ses déterminations en faisant fi de son désir et de sa liberté, il ne peut pas non plus pour autant refuser toute objectivation. Le défi est celui d'une conception juste du sujet singulier et réflexif qui lui permette de comprendre les dynamiques relationnelles où il est impliqué, en les objectivant mais sans les réifier, en les assumant dans des actions de collaboration avec d'autres et respectueuses de l'altérité. Là encore, on ne voit pas comment l'on pourrait formuler un tel agir communicationnel (Habermas) de la personne sans l'anthropologie philosophique ou sans l'ethnologie avec ses astucieuses méthodes d'observation participante, encore renouvelées actuellement.

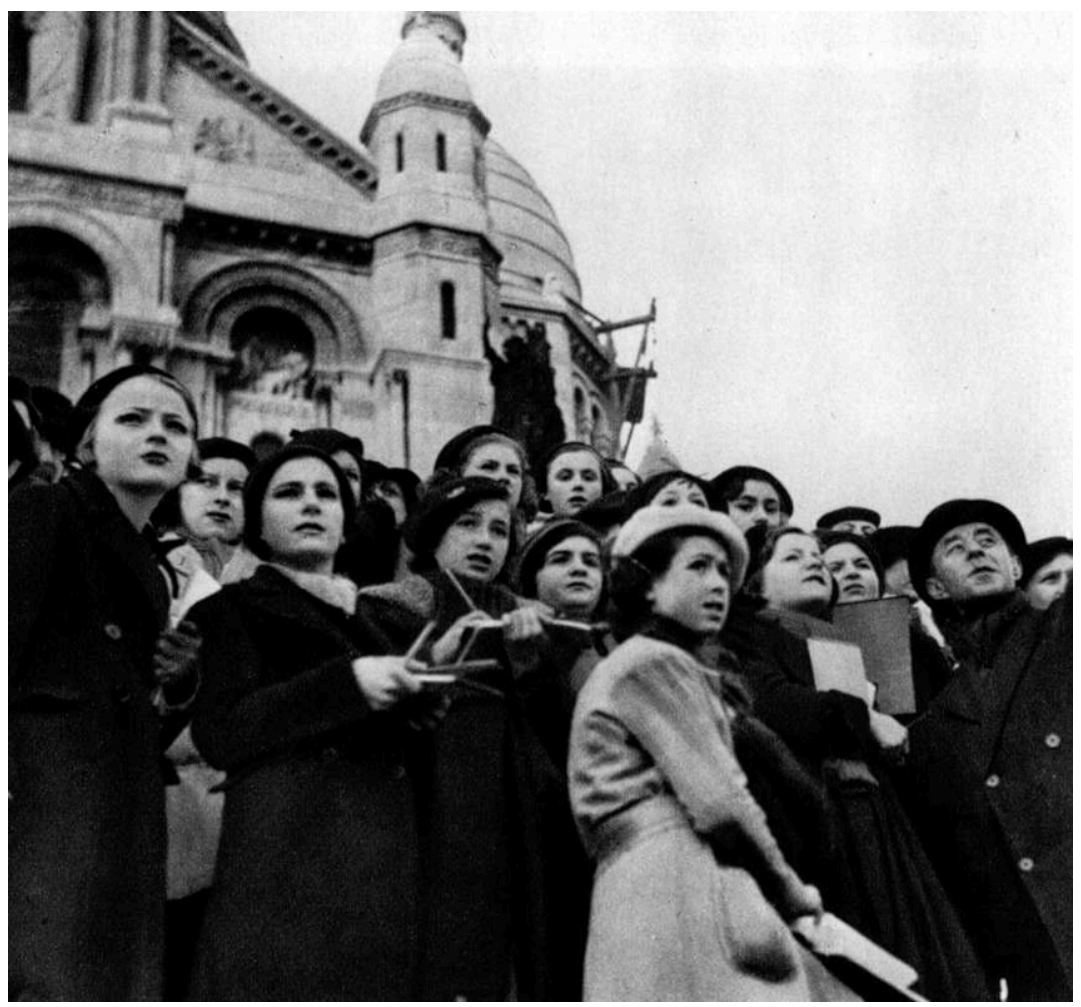

Une classe-promenade à Paris. L'histoire d'un quartier. Image (légèrement réduite) tirée de : L'enfant d'âge scolaire / P. Barrier.-Paris : Horizon de France, 1937.-« Le visage de l'enfance ».-p. 293 


\section{Ces défis sont les tremplins de l'approche anthropologique}

formation et les approches anthropologiques.
fondez-vous est donc historique entre

\section{III.1. De la déconstruction anthropologique de la violence et du sacré à une ritologie des personnes}

Le rite, dont le modèle est le sacrifice (sacer-facere), est un acte ou une série d'actes, réglés par une tradition et accomplis selon la forme et les protocoles adoptés par la collectivité ou une autorité reconnue. Il met en relation les hommes entre eux et avec une transcendance (dieux mais aussi mythes, textes, valeurs, concepts), par l'intermédiaire d'une victime ou d'une offrande. Pour Mauss, l'opposition sacré / profane est illusoire "si les dieux, chacun à son heure, sortent du temple et deviennent profanes, nous voyons, par contre, les choses humaines mais sociales, la patrie, la propriété, le travail, la personne humaine, y entrer l'une après l'autre $»^{19}$ ? Louis Dumont montre magistralement comment l'on passe d'une société traditionnelle basée sur le sacré archaïque (Homo hierarchicus ${ }^{20}$ de hieros le sacré en gr.) qui sépare en castes pour éliminer la violence rivalitaire et favoriser la communion holistique à une société moderne des individus (Homo aequalis ${ }^{21}$ ) où la dérégulation des rites et des interdits de la violence institutionnelle et sacrale antérieure au nom de l'égalitaire s'emballe dans un égalitarisme indifférenciateur qui déchaîne la violence endémique. René Girard ${ }^{22}$ permet de comprendre encore plus précisément: les rites ne sont pas des pratiques étranges et superstitieuses mais des efforts raisonnables pour fonder un consensus en stoppant le conflit de tous contre tous. Les hommes, dans les sociétés archaïques et sacrales $^{23}$ régulent la violence endémique qui sourd en permanence des mimétismes de la relation en la canalisant contre un ou quelques-uns, par des rites qui rappellent le mécanisme de bouc émissaire réconciliateur. Le rite sacrificiel, commémore cette éviction fondatrice qui solidarise la communauté et permet la culture. Le sacré archaïque différencie et ségrégue les personnes pour éviter la violence, par un sacré qui la contient à tous les sens du terme. Les sociétés modernes et individualistes critiquent, à juste titre, au nom de la liberté et de l'égalité, le sacré traditionnel qu'elles refoulent. Mais, ce faisant, elles méconnaissent la fonction et l'utilité des rites et des interdits : elles les dissolvent au lieu de se contenter de les transformer dans le sens d'institutions plus justes, déployant par là ce qu'ils ne peuvent plus contenir, à savoir la violence endémique et indifférenciatrice qui submerge le social. La solution pour sortir de la violence apparaît donc au-delà de la déritualisation actuelle, non comme une régression nostalgique vers le sacré archaïque, mais comme la recherche d'un sacré non violent avec des rites respectueux des personnes et des Droits de l'Homme.

L'école et le langage, comme toutes les institutions humaines, obéissent aux lois du système sacrificiel : elles sont constituées de rites, d'interdits et de jeux qu'il importe d'observer, sans les dénier, mais de les critiquer et de les transformer. Prisonnières du tabou, les disciplines qui étudient l'éducation comme le langage, parviennent très difficilement et progressivement à lever la méconnaissance qui pèse sur le sacré. 
quand on connaît l'importance du jeu dans l'éducation, lorsqu'on sait que seuls les interdits et les rites de passage avec leurs épreuves initiatiques (plus ou moins violentes) permettent la genèse d'une identité personnelle et sociale, que la transmutation des percepts et des affects en concepts s'opère grâce au ciment des mythes et des rites (Durkheim), on a du mal à accepter un tel refoulement irrationnel de la part des sciences de l'éducation. Aude sapere! Il est grand temps d'assumer l'étude du sacré dans l'éducation comme dans toutes les institutions du social. Si la critique de la violence, le souci de la vérité scientifique, de la liberté et de la dignité de la personne, comme valeurs fondatrices d'une épistémologie moderne, nous guident, nous n'avons pas à redouter les régressions archaïques que l'étude du sacré serait susceptible de nous faire encourir.

\section{III.2. L'interstructuration du social et des significations cognitives}

Ce sont toujours les mêmes catégorisations d'un sacré archaïque ségrégateur sur le plan de la réflexion épistémologique qui interdit de relier des champs, entre les disciplines comme entre les domaines de la pensée. L'opposition entre instruction et socialisation empêche de comprendre pourquoi et comment la cognition (comme compréhension et production de significations) ne s'opère que dans certains dispositifs interlocutifs et discursifs entre les partenaires de la relation d'enseignement / apprentissage. La communication n'est pas seulement un moyen de transmission d'informations préalablement constituées (mentalisme) elle est surtout l'espace où se construisent et où circulent des significations entre les interlocuteurs. La capacité de comprendre et de produire des significations symboliques dépend toujours de la capacité de participer à des espaces discursifs et interlocutifs. L'appropriation des significations et des notions conceptuelles des différents domaines disciplinaires du symbolique dépend des modalités de la relation d'enseignement / apprentissage (Vygotski ${ }^{24}$, Bruner ${ }^{25}$ ) tout autant que des relations inscrites dans les postures interlocutives des différents domaines et champs du savoir avec leurs pratiques et leurs genres discursifs (Bakhtine $\left.{ }^{26}\right)$.

Enfin, c'est dans et par la communication avec ses interactions que se construisent et s'actualisent les significations symboliques autant que les partenaires de la communication eux-mêmes dans leur identité subjective. Et c'est enfin dans le même temps que s'opère leur commune redéfinition du cadre relationnel et institutionnel où leur échange langagier ${ }^{27} s$ 'inscrit (Francis Jacques ${ }^{28}$ ).

51 En fait, dans l'échange symbolique et langagier, précisé et organisé par les dispositifs interlocutifs des rites et des genres des différents domaines du symbolique, se coconstruisent conjointement les significations, les partenaires de l'échange autant que le quotidien lui-même avec les (ré) interprétations des rôles et des places dans l'institution.

52 Ainsi donc, les trois grandes principales finalités de l'éducation, sont simultanées et interfaces dans l'échange en classe : c'est en co-construisant les savoirs qu'on permet une socialisation citoyenne et démocratique et qu'on autorise l'émergence de l'élève comme sujet et personne.

53 Il a fallu beaucoup de temps pour sortir de la dichotomie ségrégative entre ces différentes finalités que des approches instrumentalistes et positivistes des didactiques 
ou des autres disciplines de l'éducation verrouillaient. Ce n'est que dans les années 1980, par la conjonction du socio-cognitivisme, de l'approche dialogique de la littérature, de l'interactionnisme symbolique et de l'anthropologie interlocutive des actes de langage, qu'on commence à sortir de l'interdit de relier ces champs. La didactique du langage a attendu le milieu des années 90 pour esquisser enfin ce tournant, qui n'a pu s'exprimer vraiment ${ }^{29}$ que très récemment. Il pourrait s'approfondir encore sans doute avec le soutien actif de l'approche anthropologique du langage et de l'éducation.

\section{III.3. L'émergence interlocutive du sujet réflexif}

C'est bien la même capacité de faire des liens sans confusion entre les domaines et les notions qui est en cause dans la perception juste du sujet. Le sujet émerge grâce à la relation de signification avec l'autre et par la médiation du langage : ce qui était dans l'espace interpersonnel apparait dans l'intrasubjectif (Vygotski). C'est en assumant le langage qu'il peut se conférer à lui-même une force ontologisante qu'il puise dans le langage (Benveniste). Il ne peut se développer dans sa liberté que dans et par les contraintes du langage et grâce à l'assomption ${ }^{30}$ des codes du symbolique. Mais si son désir investit le langage, c'est parce que l'autre dans l'entreprise interlocutive, l'investit aussi. Comprendre cela demande de voir la relation triadique qui relie l'intersubjectivité des usagers des signes aux significations. La sémiologie de Peirce, autant que l'anthropologie girardienne de la mimesis désirante, nous permettent enfin de voir et de dire clairement, cette relation triadique entre les significations et les usagers des signes.

55 Au-delà encore, le sujet n'accède pleinement à la réflexivité de la personne ${ }^{31}$ (celui qui a non seulement intégré le tiers du code symbolique, mais l'autre comme tiers d'une altérité plus radicale) que par l'assomption de la loi morale (Kant).

En s'analysant dans ses pratiques symboliques avec d'autres, il peut se comprendre " soi-même comme un autre $»^{32}$ et se développer par un retour rétrospectif et prospectif conscient sur soi (pronom réflexif) et sur l'autre. On voit alors le lien ${ }^{33}$ qui s'opère entre les approches anthropologiques des sciences sociales et celles de l'anthropologie philosophique. Alors, le sujet peut se percevoir (lui-même ainsi que l'autre) dans un agir communicationnel (Habermas) qui transforme leur commune existence « dans le souci de soi, de l'autre et de l'institution juste $»^{34}$. Si nous voulons relier les savoirs à un sens plus existentiel, si nous voulons sortir de la différenciation sacrale et ségrégative de l'école non démocratique ${ }^{35}$, si nous voulons sortir de l'indifférenciation de l'école massificatrice déritualisée et anomique (Dubet), si nous voulons permettre une différenciation des élèves dans leur singularité qui ne les coupe pas d'une relation de solidarité et de coopération avec les autres, alors il faut qu'ils émergent du processus éducatif comme sujets réflexifs ou personnes.

Il en est de même pour les enseignants de plus en plus appelés à travailler avec des classes et des élèves très hétérogènes, dans le cadre d'équipes pluricatégorielles et de communautés éducatives avec d'autres radicalement différents (enseignants mais aussi soignants et parents). Comment sans une anthropologie de l'agir communicationnel de la personne former des praticiens réflexifs, capables d'inventer un métier de plus en plus 'impossible' selon le mot de Freud, c'est à dire pris dans des contradictions 
indépassables tant qu'on ne passe pas, comme le recommandait Bateson, à un niveau logique et anthropologique supérieur?

Les enjeux deviennent des défis lorsque des approches (ensembles cohérents de points de vue, perspectives, regards, d'outils conceptuels et notionnels, de modèles, de démarches théoriques et méthodologiques) apparaissent comme des stratégies averties et outillées pour discerner les impasses à éviter ou au contraire les objectifs à atteindre selon certaines conditions. Là où d'autres ont échoué, certaines approches découvrent leur pertinence et leur identité : ainsi vont les (r) évolutions scientifiques.

\section{Présentation du dossier}

59 Deux numéros successifs de la Revue Tréma (23 et 24) seront consacrés à l'essor de l'approche anthropologique en éducation et en formation. Ce premier numéro ciblera plutôt les aspects relationnels, interlocutifs et rituels de la relation éducative. Sans négliger leurs effets sur le rapport aux savoirs et sur la socialisation, il explorera surtout ceux qui concernent l'émergence de l'élève comme sujet de l'apprentissage ou de l'enseignant comme sujet d'une pratique réflexive (Perrenoud).

60 Le second numéro, sans cesser d'étudier les fonctionnements de l'interlocution entre les partenaires de la relation d'enseignement-apprentissage ou de formation avec l'impact sur les identités subjectives singulières et professionnelles, se concentrera davantage sur les enjeux anthropologiques des savoirs des différentes disciplines dans leur transversalité et leurs spécificités.

\section{La relation éducative dans ses fondements et ses particularités}

61 Jacky Beillerot conseille de prendre en considération la médiation, si l'on veut que la relation ne soit pas simplement duelle mais triangulaire. Pour cela, il affronte l'ambiguïté et la polysémie de la notion de médiation en proposant une mise à plat des différents usages qu'elle rencontre aujourd'hui dans l'éducation.

62 Si le concept a été travaillé par la philosophie et même la théologie, aujourd'hui c'est surtout au droit qu'elle emprunte ses principaux traits d'arbitrage et de régulation par traitement alternatif des conflits. Sans laisser dans l'ombre les aspects de médiation ou rémédiation cognitives, essentiels dans la fonction de l'enseignant qui doit assumer sa place de tiers dans la difficile triangulation didactique et pédagogique, l'auteur souligne l'ambivalence des pratiques de médiation sur le plan social.

63 La carence actuelle de médiations dans le social peut toujours favoriser la déliaison ou la rupture du lien social, pour les individus comme pour les communautés et le recours aux diverses instances de médiation est précieux, mais délicat. Les dérives de la médiation, qu'elle soit intra-institutionnelle (dans la vie de classe) ou interinstitutionnelle (entre l'école et les familles), sont redoutables car elles peuvent consacrer les hostilités, communautaristes ou autres, par des collusions contre ou au contraire étouffer tout conflit dans des mystifications consensuelles totalitaires. Le concept clé de médiation demande alors une approche relationnelle et rationnelle exigeante, l'analyse du rôle du tiers et de son intégration est indispensable pour éviter le retour de la violence ou sa perpétuation. Il s'agit toujours d'éviter les confusions sans 
ignorer les paradoxes. L'auteur nous renvoie alors aux intéressants travaux d'Eirick Prairat, qui prennent pour modèle l'anthropologie du sacré. Selon ce dernier, en effet, s'il faut veiller à une certaine désacralisation de l'institution dans ses aspects inégalitaires, il ne faut pas déritualiser l'éducation, il est grand temps au contraire de promouvoir une «nouvelle ritologie ${ }^{36}$ de l'école ».

Isabelle Vinatier aussi explore la puissance de la relation lorsque celle-ci est conçue de manière maîtrisée dans la recherche la formation et dans la pratique des enseignants. L'article décrit un dispositif de formation et de recherche autour de l'analyse des pratiques et montre les retombées de son efficacité transformatrice sur les situations d'enseignement en classe vécues par les participants.

Dans un dispositif interlocutif précis et exigeant sur les enjeux relationnels, chacun peut alors verbaliser son expérience, fournir des données et des traces objectivables de l'action et des situations, grâce au regard de l'autre et sur l'autre (en tant que pair ou que chercheur). Si la dynamique est dégagée de compétition rivale et du jugement qui chosifie plus qu'il n'objective, les participants parviennent à se distancer de leur vécu et à s'engager avec les autres dans une co-analyse productive. praticiens réflexifs et débouchent, par un effet en abîme et en miroir, sur l'amélioration des compétences en classe. C'est pour eux et pour leurs élèves "une plus value de connaissances sur la situation permettent au professionnel concerné d'augmenter ses capacités d'action ». Au fur et à mesure des séances, la parole échangée se fait plus efficace et se répercute sur l'invention des dispositifs et des situations en classe. Le collectif parviendra à analyser les pièges interactionnels de la relation d'enseignement où par la captation et l'instrumentalisation de l'enseignant, par les rivalités entre élèves, s'exclut le savoir comme tiers. Les participants parviendront à partager avec les élèves cette prise de conscience des phénomènes interactionnels et à provoquer des transformations importantes : « pour chaque professeur, (de) recouvrer son autonomie et (de) mettre les élèves en position de conquérir la leur».

Sur le plan méthodologique les travaux puisent aux sources conjointes de la démarche d'analyse de pratiques de Marguerite Altet (2000), de l'auto-confrontation croisée, développée en clinique de l'activité par Theureau et ils s'inspirent de l'anthropologie interlocutive de Francis Jacques.

Michel Tozzi réfléchit aux enjeux éducatifs, sociaux et anthropologiques de l'actuelle montée en puissance du débat comme genre dans l'institution scolaire. Celui-ci est désormais recommandé et promu par les Instructions officielles, dans la vie scolaire, dans la vie de classe et même comme genre didactique par différentes disciplines (philosophie, mais aussi littérature, éducation civique, etc.). A cette faveur du débat, à la fois comme objet d'étude et comme médium d'apprentissage, il voit de grands avantages, car le débat est un dispositif relationnel et une méthodologie interactionnelle de partage de la parole dont les visées sont multiples. Les bénéfices de la pratique du débat sont non seulement langagiers (compétences orales, discursives et 
communicationnelles) mais aussi cognitifs : une co-construction des significations et des savoirs devient possible. Enfin ils sont sociaux et politiques : on sort de la volonté de séduire, d'influencer de con-vaincre l'autre pour entrer dans un agir communicationnel démocratique de partage de la construction de l'institution.

Il souligne aussi les aspects éthiques de cette méthodologie du débattre. On découvre une éthique de l'altérité qui demande de prendre en considération l'autre dans sa corporéité, son visage (Lévinas), ses affects, et de «faire avec » les différences radicales et imprévisibles qu'il nous offre. Ce réglage du parler-entre et de l'inter-dire, nous amène à surseoir à notre impatience, à dominer notre désir de toute puissance, vers la redécouverte profonde de l'interdit de la violence. L'enjeu du débat alors est non seulement celui de la démocratie mais celui de l'hominisation. Pourtant le débat ne parvient à la hauteur d'une telle éthique de l'autre qu'à condition de respecter une éthique de la pensée : celle-ci implique le respect de la parole en commun avec celui de la rigueur logique de l'argumentation et des contraintes thématiques mais elle demande aussi d'oser interroger les préjugés, de critiquer les convictions et les dogmes. Pour cela, les enseignants devront éviter de refléter en classe, les pratiques du débat démagogique et manipulateur de la scène médiatique. Dès lors, le débat argumenté ne peut condamner à un perspectivisme relativiste voire cynique puisqu'il demande la pratique d'une valeur fondamentale : l'intégration du tiers, le tiers symbolique (visée logique et coopérative) et le tiers personnel, celui de la personne de l'autre.

Dans son article Jean-Marie Labelle regrette l'insuffisante prise en compte habituelle de la relation et de son action, tant dans l'éducation que la formation et même la recherche. La rencontre éducative, en effet, n'est pas une simple circonstance du rapport au savoir, mais elle participe directement à la conduite épistémique, il en est de même pour la formation et pour la recherche.

Cela demande une conception non triviale de la réciprocité : en faire un véritable concept et observer comment agit la relation dans les deux sens d'une véritable mutualité. "La référence à la réciprocité éducative invite à prendre résolument en compte le « vivre ensemble quotidien» où s'enracine pour l'apprenant la fabrication de son savoir, et, pour le chercheur l'élaboration des connaissances".

Des sciences de l'éducation qui excluraient le sujet en action et en interaction, constitueraient alors un obstacle majeur. A la suite d'auteurs comme Morin, Todorov, Ricœur, ou Charles Taylor, il en appelle alors à une nouvelle approche épistémologique, plus proche de l'art, qui reposerait enfin sur la conception d'un sujet réflexif, advenant à la conscience et à l'existence, par et pour l'autre, dans la parole échangée.

73 Loin de condamner au flou méthodologique, la démarche au contraire permet d'élaborer des dispositifs de recherche très précis. On n'a plus la classique opposition entre méthodes expérimentales et cliniques, mais une démarche de recueil des données qui s'apparente à la minutie du chercheur d'or et des analyses de discours qui engagent de véritables textes où l'élaboration de la connaissance croise un agir professionnel et relationnel de la personne comme soi réflexif.

74 La méthode microspective et expérienciée formalisée par l'auteur et dont il indique ici les procédures vient alors rejoindre l'ethnométhodologie, l'entretien d'explicitation de Pierre Vermersch, la démarche microhistorique de Carlo Ginzburg, dans la riche panoplie de l'approche anthropologique. 


\section{Corps, rite, culture et valeur : repenser l'universel et le particulier}

d'enseignement. Il s'appuie sur son expérience de formation d'enseignants à travers les modules corps et voix. Qu'on le veuille ou non, les corps dans la classe sont là, ils participent pleinement au processus cognitif comme à la mimesis désirante de l'enseignement-apprentissage. Si l'enseignant doit faire signe et permettre l'entrée dans la signification à travers sa parole, sa corporalité et gestualité en seront les principaux supports et vecteurs. Le corps de l'élève aujourd'hui dans les Instructions officielles est heureusement l'objet d'une attention plus respectueuse, mais le corps de l'enseignant reste impensé si ce n'est à travers la norme, or il peut se faire opaque et obstacle au lieu d'être le véhicule de l'affranchissement de l'élève vers l'appropriation des savoirs.

a mimesis désirante d'apprentissage peut buter sur l'obstacle de la séduction et de la rivalité conflictuelle, mais elle peut aussi déraper lorsque l'enseignant dénie, évacue ou sublime excessivement le corps. Cette dénégation participe alors à la violence symbolique et l'affrontement culturel avec les élèves est inévitable. L'écart grandissant sur le plan des normes socio-culturelles entre les enseignants et la majorité de leurs élèves oppose alors des ethnocentrismes de classe. Chacun des partenaires de la relation éducative, tels Héraclès et Eurysthée les personnages du mythe (analysés par Serge Boimare comme exemples de la confrontation de l'enseignante au refus d'apprendre) est alors amené à se caricaturer dans sa corporalité : tandis que l'un «tente d'étouffer le mental sous la carapace corporelle » l'autre en symétrique « essaie de plier le corps sous le joug de l'esprit ».

L'auteur recommande alors aux enseignants de ne plus négliger le travail sur leur corporéité en situation d'enseignement, il se réfère pour cela aux avancées de la didactique de l'EPS mais aussi à l'apport des pratiques ritualisées du jeu dramatique ou de l'Aïkido Kobayashi.

Pour Marie-Louise Martinez-Verdier les enseignants peuvent partager avec les élèves, même très jeunes, une véritable déconstruction anthropologique de la violence éducative et, par cette compréhension, dépasser la violence et inventer en classe, une éducation des personnes dans la coopération et l'ouverture à l'altérité ... Ces valeurs, avec une présentation fine des rites de passage et de leur enjeu violent, sont lisibles par exemple, dans un album jeunesse de grande qualité littéraire: Yacouba, publié chez Seuil Jeunesse.

Cet album de Thierry Dedieu réussit en quelques pages, par une iconographie d'une rare qualité, quelques sobres trouvailles littéraires, une écriture d'un grand classicisme, à dévoiler les mécanismes de la violence et du sacré et les grandes solutions anthropologiques pour sortir du cycle infernal du désir mimétique, de la rivalité et du bouc émissaire. L'essentiel d'une grande expérience éducative est là, confié dans un langage accessible aux jeunes enfants dans un genre qui va du récit initiatique au roman d'éducation pour atteindre par moments la dimension d'un conte philosophique. L'anthropologie, sans dire la vérité de la littérature qui se suffit à elle- 
même en touchant l'affect autant que l'intellect, peut en souligner les grands traits et la cohérence.

L'enseignant, à condition qu'il soit informé et formé selon l'approche anthropologique (concepts de l'anthropologie du sacré : rite, fête, mythe, indifférenciation, désir et rivalité mimétiques mais aussi concepts de l'analyse interlocutive: opposition entre récit et discours, locuteur, interlocuteur, sujets interlocutifs, non-personne du délocuté, processus interlocutifs de personnification, etc.) peut ouvrir aux élèves l'accès au sens profond des textes. Il peut alors mettre en place, dans la pratique de la classe, des rites alternatifs et démocratiques, comme le débat. Ici, le dilemme et le discours (entre deux personnages, mais aussi intériorisé chez le personnage principal) sont des procédés littéraires qui permettent de montrer l'émergence de la conscience et de la subjectivité réflexive. Mais le débat peut aussi devenir un genre d'apprentissage (débat d'interprétation littéraire) croisant la relation au texte et la relation intersubjective pour permettre l'émergence interlocutive de l'élève comme personne. Dès lors, la notion de personne apparaît comme susceptible de refonder le sujet de l'éducation autour d'une valeur partagée, comme sacré non violent et laïque (Droits de l'Homme, Instructions officielles, etc.).

Anne-Marie Drouin-Hans considère qu'il importe vraiment de dissiper certaines confusions dans la formation des enseignants, particulièrement dans ce qui touche aux valeurs de l'éducation et à la laïcité. Si la relativisation des cultures et de leurs coutumes est l'apport précieux de l'anthropologie, depuis Montaigne, Pascal jusqu'à Levi-Strauss, elle ne met pas pour autant en péril la recherche de l'universel en l'homme, particulièrement sur le plan moral et éthique. La mentalité actuelle confond dangereusement les domaines et les notions : on voudra mondialiser et universaliser certaines pratiques culturelles de l'occident («domination économique, confort matériel onéreux, débauche, etc.»), comme on relativisera certains de ses principes ("liberté, démocratie, développement des sciences, etc.»). Inversement, dans certains discours scientifiques, l'esprit post-moderne est prêt à légitimer au nom du respect de l'autre " les cultures de la violence ", avec leurs pratiques d'intimidation et leur organisation sociale inégalitaire.

Pour l'auteur, l'approche anthropologique est salutaire à condition qu'on en fasse bon usage et surtout qu'on redonne aux concepts leur pertinence " la position relativiste qui fusionne valeur et culture est une façon d'éluder la question des valeurs » et "parler des valeurs n'évoque pas les valeurs boursières, changeantes selon les caprices de l'économie, mais l'idéal du bien en quête d'éternité et d'universalité ".

Elle propose alors pour guider l'indispensable travail de discernement, l'utilisation de la notion d'universalisable (qu'elle reprend à Selim Abou) et la notion d'universel négatif qu'elle emprunte à Catherine Larrere.

Ce faisant, elle donne des indications sur le sujet d'un agir communicationnel respectueux de l'altérité en éducation. L'ouverture à l'autre et la tolérance ne peuvent se vivre que dans la réciprocité et la réflexivité du souci de l'autre et de soi-même 
comme personne: "Si le tout autre est aussi ce qui ne tolère pas le différent, reconnaître l'autre c'est se nier soi-même».

Dans la convergence et la cohérence, malgré leurs références plurielles, ces différents articles montrent tous l'enjeu actuel d'un tournant et d'un paradigme anthropologiques dans l'éducation et la formation.

Le sacré traditionnel avec ses rites et ses interdits très forts avait le mérite de favoriser le partage des mêmes repères et valeurs dans l'école, mais leur inconvénient inacceptable pour nos consciences modernes est l'hétéronomie et la différenciation ségrégative du social. Pourtant la déritualisation de la crise actuelle ne nous a pas donné l'autonomie et la démocratisation attendues. On constate une grande indifférenciation (confusion, tant au niveau des règles de la socialisation, que de l'appropriation des savoirs, ou de l'identité des élèves), la massification actuelle qui n'est pas encore véritable démocratisation a provoqué un désordre anomique. Comme souvent dans l'histoire, le désordre occasionne le retour régressif à une sacralisation violente et ségrégative : cycle de la violence et du sacré. L'approche anthropologique ne se contente pas de déconstruire ces phénomènes dans l'éducation, par ses démarches et ses outils scientifiques pris dans les sciences sociales, les sciences du langage ou la philosophie interlocutive du langage, elle montre qu'on peut insuffler de nouvelles dynamiques relationnelles, rituelles et interactionnelles démocratiques.

Le débat, les dispositifs de médiation, les analyses de pratiques, les approches intersubjectives et participatives en formation, nous donnent aujourd'hui des exemples de rituels non violents qui peuvent inciter à un véritable agir communicationnel de la personne dans l'enseignement, la formation et la recherche.

\section{NOTES}

1. Bourdieu Pierre, 1997, Méditations pascaliennes; Seuil : tout au long de son oeuvre l'auteur rappelle qu'il se définit lui - même autant comme anthropologue que sociologue.

2. Les représentants de cette discipline sont nombreux et se réfèrent à des théories diverses, on peut citer parmi eux quelques noms représentatifs (Lévinas, Ricœur, Habermas, Jacques, etc).

3. C'est le cas par exemple dans les travaux de Bourdieu sur l'école, avec les rites d'institution et les processus de la violence symbolique. Voir par ex. Pierre Bourdieu, «Les rites d'institution »; in Actes de la recherche en sciences sociales, $\mathrm{n}^{\circ} 43$; 1982 ou encore (avec Loïc Wacquant) : Réponses; Pour une anthropologie réflexive ; Seuil ; 1992.

4. On en trouvera aussi la remarque dans le $\mathrm{N}^{\circ} 31$ de la Revue Spirale (laboratoire des sciences de l'éducation de Lille) consacré à la question " Anthropologie de la formation et de l'éducation », et particulièrement dans l'article de Jean - Louis Legrand «Place de l'anthropologie dans les sciences de l'éducation en France », pp. 5 - 19, qui recense les inscriptions dans l'annuaire de l'AECSE (principale association avec l'AFIRSE) d'enseignants chercheurs en sciences de l'éducation.

5. Un paradigme est un ensemble cohérent et convergent de disciplines et de théories dont les concepts et les démarches semblent conciliables entre elles (Kunh Thomas (1962 et trad. 1970); La structure des révolutions scientifiques ; Flammarion. 
6. Selon Kant, en effet, l'homme ne peut devenir homme que par l'éducation.

7. Voir ici par exemple les travaux d'Yves Michaud sur la violence qu'il caractérisait en 1986 (La violence, PUF) de concept flou et d'illusion.

8. Voir ici Robert A. D. et Terral H., 2000, Les IUFM et la formation des enseignants aujourd'hui ; PUF.

9. Aries Philippe, 1973, L'enfant et la vie familiale sous l'ancien régime, Seuil ; 1992 ; « L'enfant : la fin d'un règne ", in Finie la Famille?, Paris Autrement, série Mutations poche ; 8.

10. Kant Emmanuel (1993, VIIe éd.), Réflexions sur l'éducation, Vrin.

11. Kant Emmanuel (1785, trad.Victor Delbos revue par A. Philonenko, Vrin, 1992), Fondements de la métaphysique des mours: dont l'impératif pratique "Agis de telle sorte que tu traites l'humanité aussi bien dans ta personne que dans la personne de tout autre, toujours en même temps comme une fin, et jamais simplement comme un moyen", IIe section p. 105, nous rappelle que toutes les finalités ne peuvent jamais être qu'au service d'une seule fin de l'éducation.

12. Voir, 1999, La nouvelle Revue de l'AIS, $\mathrm{N}^{\circ} 4$ et $\mathrm{N}^{\circ} 5$ "Sujet et personne " coordonnés par Martinez Marie - Louise.

13. Perrenoud, Philippe, 2001, Développer la pratique réflexive dans le métier d'enseignant; ESF.

14. Voir par ex. Legrand, Jean - Louis (op. cit.) «Des objets tabous en sciences de l'éducation? Dès qu'on descend à une occurrence (sur un total de 1464) cela est quasiment insignifiant.(. ..) les thématiques qu'on ne retrouve qu'une seule fois : échange, filiation, initiation, mémoire collective, mythe, pouvoir... (...) aucune occurrence parmi les objets de recherche recensés dans l'index de l'annuaire : croyance, dieux, don, ... (...) islam, islamisation, mort, religion.... Il en est de même de tout ce qui touche au religieux et au spirituel. Hormis deux entrées hindouisme et soufisme, l'un par un professeur émérite, l'autre par un chercheur hors statut, je n'ai trouvé nulle trace... (...) tout se passe comme si ces questions étaient elles aussi tabous. On peut se demander si elles ne le sont pas encore plus qu'il y a un siècle où l'un des illustres titulaires de la chaire de Sciences de l'éducation à la Sorbonne, Durkheim, travaillaient sur les formes élémentaires de la vie religieuse. (...) En 2001, la brochure de l'Association est totalement remaniée et l'anthropologie a, du point de vue langagier, une place plus importante. » pp. 13 - 16.

15. Durkheim Emile, 1979, Les formes élémentaires de la vie religieuse; PUF.

16. Mauss Marcel, 1968, Fonctions sociales du sacré; Ed. de Minuit ; p. 5.

17. Voir Les travaux d'Agnès Van Zanten, par ex. « De la diversité à la ségrégation scolaire »; $L a$ nouvelle revue de l'AIS ; Marginalisation, intégration ; N 8 ; 1999 ou encore de Georges Felouzis «La ségrégation ethnique au collège et ses conséquences »; Revue Française de sociologie; juillet septembre, 2003.

18. Milner Claude, 1983, De l'école, Fayard.

19. Mauss Marcel, 1968, Fonctions sociales du sacré ; ed. de Minuit.

20. Dumont Louis, 1966, Homo Hierarchicus ; le système des castes et ses implications ; Gallimard

21. Dumont Louis, 1977, Homo aequalis ; Gallimard ; 1983, Essais sur l'individualisme ; une perspective anthropologique sur l'idéologie moderne; Seuil.

22. Girard René, 1972, La violence et le sacré, Grasset ; et, 1982, Le bouc émissaire ; Grasset

23. L'adjectif sacral, ici, dans un sens négatif de sacré fondé sur le sacrifice violent et la ségrégation est repris à Louis Dumont qui l'oppose à un sacré non violent et plus moderne fondé sur l'égalité des personnes, sacré que l'on retrouve dans certaines pratiques sociales religieuses ou profanes.

24. Vygotski, Lev Sémionovitch, 1885, Pensée et langage, trad. du russe par Françoise Sève ; Editions sociales.

25. Bruner, J. S, 1982, Le développement de l'enfant, savoir faire savoir dire; trad. de l'anglais par Michel Deleau ; PUF.

26. Bachtine, Mikhaill, 1984, Esthétique de la création verbale; trad. du russe par Alfreda Aucouturier et préfacé par Tzvétan Todorov, Gallimard.

27. Fait de mots mais aussi d'autres signes, de gestes corporels et d'objets inscrits dans des systèmes de signification symboliquement codifiés. 
28. Jacques, Francis, 1985, L'espace logique de l'interlocution; PUF.

29. Voir par exemple : Bucheton, Dominique et Chabanne, Jean - Charles, 2002, Parler et écrire pour penser, apprendre et se construire ; PUF ; Bernie, Jean - Paul ; sous la dir. de. 2001 ; Apprentissage, développement et significations; en hommage à Michel Brossard ; Presses Universitaires de Bordeaux et Tozzi Michel, 2001, L'éveil de la pensée réflexive à l'école primaire ; Hachette ; et d'autres ...

30. Benveniste, Emile, 1966, «C'est dans et par le langage que l'homme se constitue comme sujet : parce que le langage seul fonde en réalité, dans la réalité qui est celle de l'égo (...) est égo qui dit égo "; Problèmes de linguistique générale (Tome 1, pp. 259 - 260, Gallimard 1966).

31. Martinez, Marie - Louise, 2002, L'émergence de la personne ; éduquer, accompagner ; L'Harmattan; et, 1997, Vers la réduction de la violence à l'école; contribution à l'étude de quelques concepts pour une anthropologie relationnelle de la personne en philosophie de l'éducation; Septentrion.

32. Ricœur, Paul, 1990, Soi - même comme un autre; Seuil.

33. Certains auteurs, comme Jérôme BRUNER par exemple dans ses derniers travaux, 1990, Car la culture donne forme à l'esprit ; Eshel ; etc.) font le lien entre une psychologie sociale de l'éducation qui plonge ses racines dans l'anthropologie culturelle et l'anthropologie pragmatique du langage.

34. Ricœur, Paul idem.

35. Avec la violence symbolique de ses rites d'institution critiqués déjà par Bourdieu.

36. Voir Prairat, Eirick, 2001, «Jalons pour une ritologie scolaire », Sanction et socialisation ; PUF ; pp. $208-213$.

\section{RÉSUMÉS}

De nouveaux enjeux dans l'éducation et la formation se manifestent par des obstacles inédits devant lesquels hésitent et bronchent les approches classiques en sciences de l'éducation qui n'ont pas su, pas pu ou pas voulu les aborder. Ces résistances réclament un angle d'attaque renouvelé : autant de défis pour l'approche anthropologique qui se développe en sciences de l'éducation en un nouveau paradigme. Etudier ces enjeux (sociaux, institutionnels et épistémologiques), ces résistances (tabou du rite et du sacré, difficulté à penser ensemble la genèse cognitive et la genèse sociale, lenteur à penser l'émergence du sujet dans la relation) nous permet de comprendre quels défis devra relever l'approche anthropologique.

New deals are rising in ducation and teacher-training, with new kinds of obstacles. Usual approache on éducational sciences, are stambling on them unable to find the way how to surround them. Such hindrance claim a new approach: tath's a challenge for the new athropological approach rising on educational sciences into a new paradigme. Studying these deals (socials, institutionnals and épistemologics one), these obstacles (things sacred and ritual as forbidden subjects, difficulty to think both cognitiv and social genesis, slowness on thinking the subjectiv emergence into the relationship), shows us better the athropological approach actual challenge. 
INDEX

Mots-clés : approche anthropologique, émergence interlocutive, interstructuration, sacré, signification cognitive, social, sujet réflexif

Keywords : anthropological approach, cognitiv signification, emergence on interlocutiv process, interstructuration, reflexiv subject, sacred, social

\section{AUTEUR}

MARIE-LOUISE MARTINEZ-VERDIER

Maître de conférences en sciences de l'éducation, IUFM de Nice 